\begin{tabular}{|c|c|c|c|}
\hline Eiszeitalter u. Gegenwart & $\mathbf{2 7}$ & $\begin{array}{c}174-188 \\
7 \text { Abb., 2 Taf., } \\
1 \text { Tab. }\end{array}$ & Öbringen/Württ. 1976 \\
\hline
\end{tabular}

\title{
Rezente Periglazialerscheinungen im Gebiet des Daisetsu San in Nordjapan
}

\author{
Ludwig Ellenberg*)
}

[Periglacial Phenomena of the Daisetsu San, Northern Japan]

$\mathrm{Kurzf}$ assung: In Japan gibt es nur eine einzige Gebirgsregion, in der rezente Periglazialformen weitverbreitet auftreten. Es ist die Vulkan-Gruppe des Daisetsu San im zentralen Teil der Insel Hokkaido, der nördlichsten großen Insel Japans.

Unter Mitverwendung der Beobachtungen japanischer Autoren wird die Regelhaftigkeit des Auftretens von rezenten Periglazialformen und ihrer Vergesellschaftung in Tab. 1 aufgezeigt.

[Periglacial phenomena of the Daisetsu San, northern Japan]

A bstract: In Japan exists only one mountain region, where periglacial phenomena are widely spread. This is the volcanic group of the Daisetsu San in the central part of Hokkaido, the northern island of Japan.

Table 1 shows the local distribution and the various qualities of the periglacial forms.

\section{Einleitung}

In den Gebirgen Japans nehmen rezente Periglazialformen eine verschwindend kleine Fläche ein. Sie ist wesentlich geringer als in vielen anderen Gebirgen der humiden gemäßigten Breiten oder gar in der subnivalen Stufe innertropischer Gebirge. Für das spärliche Autreten von Formen, die durch Frostwechsel entstehen oder durch ihn mitgestaltet werden, sind mehrere Gründe zu nennen:

1. Warme und feuchte Sommer ermöglichen geschlossenen Waldwuchs bis in relativ große Höhen. Die klimatische Höhengrenze des Waldes, oberhalb der die meisten Periglazialformen entstehen, liegt in Zentral-Honshu zwischen 2400 und $2600 \mathrm{~m}$. Sie sinkt zwar gegen Nord-Honshu abrupt ab und zwar im nördlichsten Japan auf ca. 1200 m. Gleichsinnig mit ihr verringern sich jedoch auch die Maximalhöhen der Bergketten und Vulkane. Uber die Waldgrenze ragen daher in ganz Japan nur sehr kleine voneinander isolierte Bereiche auf. Die alpine Stufe steht zu der der europäischen Alpen ungefähr im Größenverhältnis $1: 10$ (Weltforstatlas 1973).

2. Die relativ kleinen Areale über der Waldgrenze sind arm an Ebenheiten und flachen Hängen, welche für die Ausprägung von Periglazialformen günstig sind. Steile Hänge und scharfe Grate herrschen vor; drei Viertel der Fläche Japans sind stärker als $15^{\circ}$ geböscht (Schwind 1967). Die Steilheit der Hänge erklärt sich bei vielen Bergen aus ihrem Ursprung als Stratovulkane von der Form des Fuji San. Außerdem sind glaziale Formen nur kleinräumig ausgeprägt. Beispielsweise fehlen Karformen an der W-Abdachung der Japanischen Alpen fast völlig. Zahlreiche Vulkane sind erst postpleistozän entstanden oder erfuhren nach den Kaltzeiten neue vulkanische Akkumulationen. Einer der wesentlichsten Gründe für die Steilheit der Hänge ist fast überall die intensive Prägung des Reliefs durch fluviatile Abtragungsprozesse, besonders im Bereich starker Hebungen und auf den Inseln südlich von Hokkaido, wo im Zuge herbstlicher Taifune intensive Regen niedergehen.

*) Anschrift des Verfassers: Dr. L. E 11 e n b e r g, Institut für Geographie der Techn. Universität Berlin, Straße des 17. Juni 135, D 1000 Berlin 12. 
3. Andesitisches Lockermaterial an den Hängen, grobblockiger Schutt und durch Denudation bis aufs Anstehende entblößte Partien sind in der alpinen Stufe häufig ausgebildet. In den Lockermassen versickert zugeführte Feuchtigkeit leicht bis in Tiefen, wo der Grund ganzjährig nicht gefriert. Die oberflächennahen Frostwechsel sind deshalb bezüglich periglazialer Formung nur wenig wirksam. Eine edaphische Ungunst zeigt sich überdies in dem spärlichen Auftreten von Moränen, wo Lockermaterial verschiedener Größenfraktionen gemischt vorliegen.

4. Die Wälder der oberen subalpinen Stufe wurden in Japan nicht, wie in vielen anderen Gebirgen der Erde, durch Weidewirtschaft zerstört und zu Grasland oder ähnlichen Vegetationstypen degradiert. Eine Almwirtschaft im europäischen Sinne gab es nirgends, schon weil das Relief dafür zu ungünstig ist. Offene, die Solifluktion nicht stark behindernde Pflanzenformationen fehlen daher auch unterhalb der klimatischen Waldgrenze fast ganz.

5. Auf der westlichen Seite von Honshu und Hokkaido, die der Japan Sea zugewandt ist, erfolgt der Einbruch des Winters meist nicht durch Fröste, sondern durch starke Schneefälle. Die Schneedecke wird rasch sehr mächtig, am Shirouma Dake z. B. mehr als $4 \mathrm{~m}$; sie mindert das Eindringen des Frostes und verhindert häufige Frostwechsel imBoden (s. z. B. HARRIS 1974). Die Schneemassen werden im Frühling trotz intensiver Schmelzvorgänge nur allmählich aufgezehrt. Daher dauert die geschlossene Schneedecke lange an, am Shirouma Dake z. B. weit länger als 140 Tage. In großen Teilen der oben erwähnten Regionen bleibt infolgedessen die frostwechselintensive Zeit des Spätfrühlings ohne Wirkung auf den Boden.

Von dem spärlichen Auftreten periglazialer Formen gibt es in Japan eine einzige Ausnahme, das Berggebiet der Vulkangruppe des Daisetsu San in Nordj a p a n. Es ist reich an Periglazialformen und sie sind hier großflächig verbreitet (Abb. 1).

Daher geriet es in den Brennpunkt der japanischen periglazialmorphologischen Forschung, die z. B. durch Arbeiten von Koaze (1958, 1965), Sako et al. (1958), Takakura

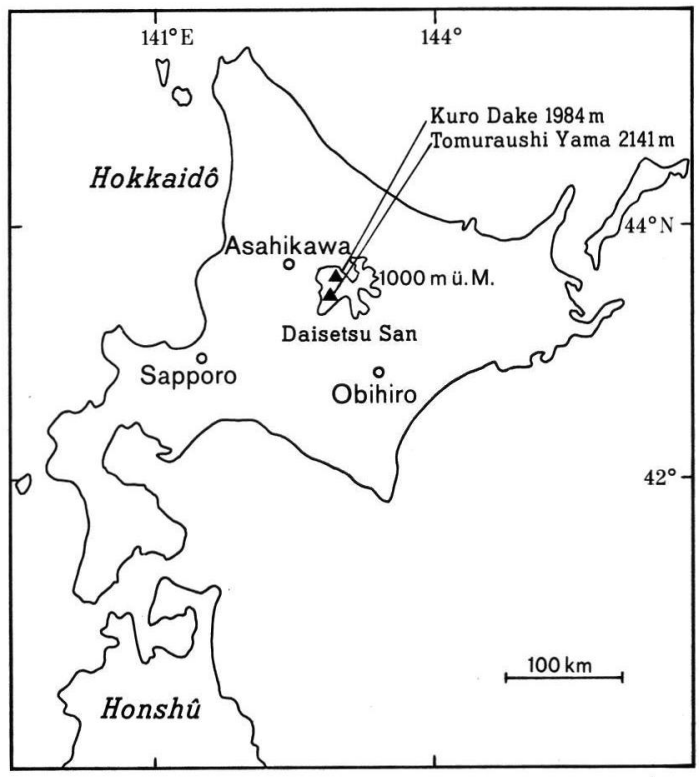

Abb. 1: Lage des Untersuchungsgebietes. 
(1972 und z. T. unveröff. Омото (1973) und Iwata (unveröff.) dokumentiert wird. Deren Befunde sollen hier durch eigene Untersuchungen ergänzt werden, die im Sommer 1973 durchgeführt wurden. Nach Beschreibung einiger Fundgebiete wird eine Aussage über die Regelhaftigkeit des Auftretens und der Vergesellschaftung von Periglazialformen im Gebiet des Daisetsu San angestrebt.

\section{Der Daisetsu San als bedeutendste Periglazialregion Japans}

Die Vulkangruppe des Daisetsu San liegt im Innern von Hokkaido zwischen $43^{\circ} 20^{\prime}$ und $43^{\circ} 50^{\prime} \mathrm{NB}$ und umfaßt ein ca. $1000 \mathrm{~km}^{2}$ großes Hochland mit weiten, nur wenig geneigten Flächen. Steilabstürzte in den Randgebieten und aufgesetzte Vulkankegel, von denen der höchste, der Asahi Dake, 2290 m erreicht, sind flächenmäßig unbedeutende Akzente. Das Gebiet des Daisetsu San bildet das Ende der Chishima-Vulkanreihe und stellt zugleich ihr mächtigstes Glied dar. Petrographisch bietet es ein reiches vulkanisches Spektrum: Laven und Basalte verschiedenen quartären Alters, Andesite, Aschen, z. T. mit Bomben und stark verfestigte Tuffe. Ausbrüche wurden in historischer Zeit nicht verzeichnet, doch ist die vulkanische Aktivität noch nicht ganz abgeklungen, wie Fumarolen und Onzen (heiße Quellen) im weiteren Umkreis des Daisetsu San anzeigen.

Warum sind im Gebiet des Daisetsu San Periglazialformen wesentlich häufiger und auf größerer Fläche anzutreffen als in den übrigenBerggebieten Japans? - Die Waldgrenze verläuft hier unterhalb von sanft geneigten Hochflächen und erreicht maximal $1850 \mathrm{~m}$ (Numuta 1972). Die alpine Stufe ist verhältnismäßig ausgedehnt und hat nur eine geringe Reliefenergie. Zwar ist auch in ihr keine starke anthropogene Degradierung der Strauchvegetation nachzuweisen. Pinus pumila bedeckt als Legföhre größere schneearme Areale und stabilisiert die oberste Bodenschicht. Doch bleiben weite Flächen übrig, die kaum oder nur spärlich bewachsen sind und in denen die Pflanzendecke Solifluktionsbewegungen nur wenig hemmen kann. Andesittrümmer, eingebettet in Tephra, treten neben Tuffen häufig auf und bilden günstige edaphische Voraussetzungen für Frostsortierungen, da Feuchtigkeit oberflächennah gespeichert werden kann und trennbare Korngrößengruppen vorhanden sind. Die mittleren winterlichen Schneeverhältnisse würden der Ausbildung von Periglazialformen zwar eher entgegen wirken, wird doch die Schneedecke im Hochwinter mächtiger als $150 \mathrm{~cm}$ und dauert länger als 160 Tage an. Die Verteilung des Schnees ist jedoch sehr unterschiedlich. Der Akkumulation an Leehängen und Mulden steht das Abblasen an windexponierten Luvhängen und Sattelflächen gegenüber, die ganzjährig schneearm bleiben.

\section{Beschreibung einzelner Gebiete ${ }^{1}$ )}

In Zusammenarbeit mit S. Iwata und K. Hirakawa konnte ich im Juli 1973 Beobachtungen im Gebiet des Daisetsu San anstellen. Beiden sei auch an dieser Stelle herzlich gedankt. Wir folgten einer Traverse von $\mathrm{N}$ nach S, ausgehend von Sounkyô (ca. $600 \mathrm{~m}$ ) über die Gipfel Kuro Dake (1984 m), Hokkai Dake (2140 m), Hakuun Dake (2230 m), Chûbetsu Dake (1963 m), Goshiki Dake (1968 m), Kaun Dake (1954 m) zum Tomuraushi Yama (2141 m), von wo nach Tomuraushi Onzen abgestiegen wurde (Abb. 2).

1) Bedeutung einiger Namen:

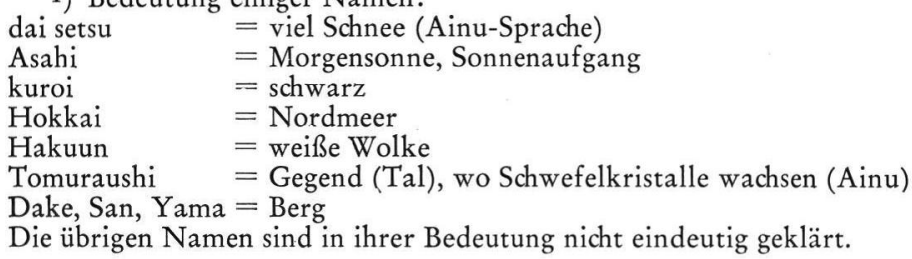




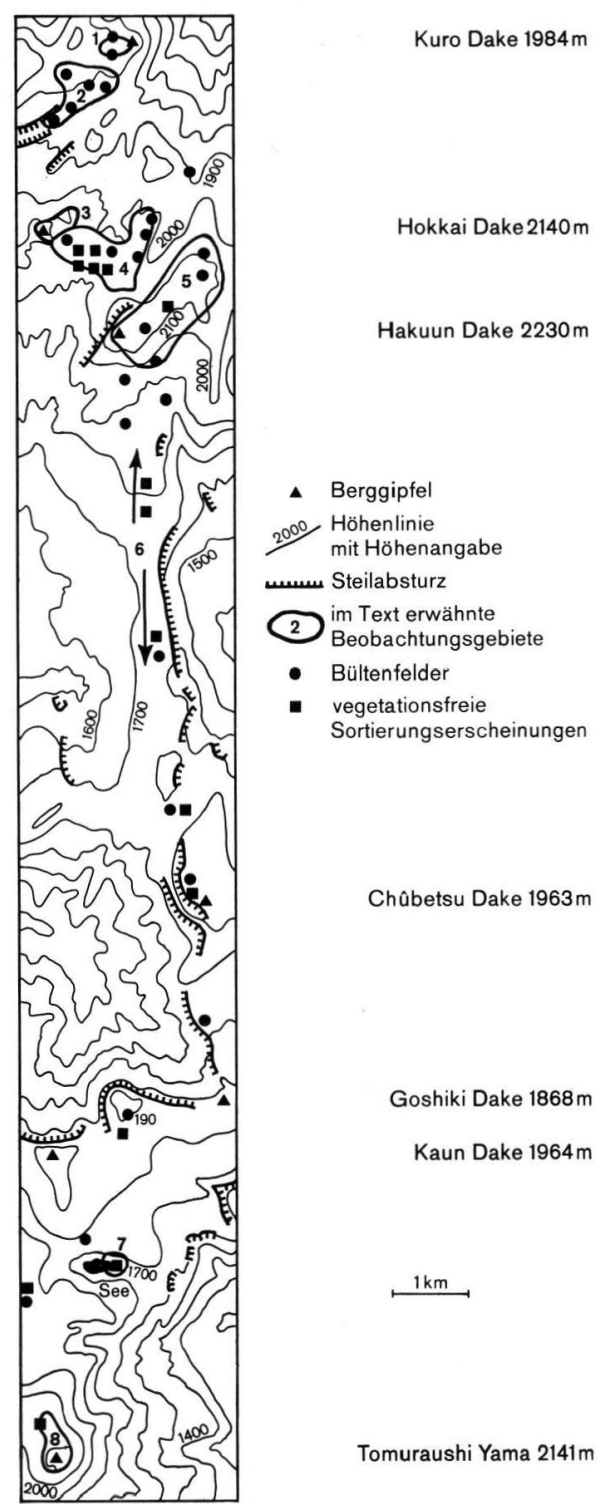

Abb. 2: Geographische Situation.

Gebiet 1: Kuro Dake

Die Hänge der $1984 \mathrm{~m}$ hohen andesitischen Kuppe fallen gegen $\mathrm{N}$ und $\mathrm{E}$ steil, gegen $\mathrm{S}$ und $\mathrm{W}$ flach $\mathrm{ab}$. An den Steilhängen, besonders in E-Exposition, ist die Pflanzendecke bis fast zum Gipfel hin dicht geschlossen. Unterhalb $1700 \mathrm{~m}$ sind es Waldbestände mit aufrecht wachsenden Picea- und Abies-Arten sowie Laubbäume wie Betula, Alnus und Sorbus, die mehr oder minder stark durch Schneedruck und Schneerutschungen verformt sind. 
Auch die Zwergbambus-Heiden (Sasa kurilensis und andere Sasa-Arten) im Unterwuchs zeigen Schneedruckformung. Über der Waldgrenze, wo sie geschlossene Bestände bilden, ist diese ebenfalls deutlich zu beobachten. Im Juli 1973 waren hier vielerorts Schneereste erhalten geblieben, auch außerhalb von Rinnen. Solifluktionsbewegungen werden durch den Sasa-Bewuchs erschwert, und Periglazialformen sind kaum ausgebildet.

Anders ist es an den flachen, etwa $10-20^{\circ}$ geneigten Hängen: stellenweise wachsen zwar Gruppen von Pinus pumila, aber der Hauptteil ist mit krautigem Carex-reichem Rasen überzogen. Hier sind an vielen Orten Treppenböden ausgebildet. Ihre Terrassenflächen(FT) sind vegetationsfrei; scherbiger Schutt ist in den obersten $5 \mathrm{~cm}$ angereichert, darunter findet man ausschließlich feines verwittertes Tephra-Material. Die Terrassenhänge (TH) sind selten höher als $30 \mathrm{~cm}$, wirken stabil und sind dicht bewachsen. Schneereste fehlten im Juli 1973. Die Treppenböden gehen in Solifluktionsloben oder in ganz überwachsene Treppenböden über, wenn die Neigung geringer wird. Diese wiederum bilden auf größeren Ebenheiten Übergänge zu Feldern mit unregelmäßig angeordneten Bülten, die maximal bis $40 \mathrm{~cm}$ aufgewölbt sind.

Gebiet 2: Schneemulden zwischen KuroDake und Hokkai Dake

Die zwischen 1800 und 1900 m hoch gelegenen Mulden haben großenteils S-Exposition. Im Winter werden hier im Lee des NW-Monsuns große Schneemassen akkumuliert. Im Juli waren die tiefen Stellen aller Senken noch schneegefüllt - mit Mächtigkeiten bis über $3 \mathrm{~m}$. An den Rändern der Schneereste in äußerst feuchtem Milieu und bei fast ebener Lage gab die Schneeschmelze Bültenfelder frei. Die bis $50 \mathrm{~cm}$ aufragenden Höcker sind meist ganz mit Pflanzen bedeckt und relativ eng geschart, $d$. h. die Rinnen zwischen den Aufwölbungen sind markant und steilwandig eingelassen. In ihnen verfingerten sich Schneereste (Taf. I, Fig. 1). Wird das Schmelzwasser bei leichter Hangneigung durch diese Rinnen gespült, kann es an ihrer Ausgestaltung mitwirken, wie mehrere Unterschneidungen von Bülten zeigen. Dies ist eine Beobachtung, die von mir auch auf anderen Bergen, z. B. dem Yôtei San in S-Hokkaido, gemacht wurde und von Fujıki (Mitt.) bestätigt wird. Frostbedingte Aufwölbung und erosive Rinnenausgestaltung gehen hier Hand in Hand. Die Kerne der einzelnen Buckel waren noch gefroren (Abb. 3), sie tauen allerdings bis Ende September auf. Die Bülten sind im Gebiet des Daisetsu San fast alle an Standorte gebunden, die im Juni ausapern. Zu dieser Zeit vollziehen sich auch die letzten Frostwechsel des Spätfrühlings im Boden, dessen oberste Schichten gut durchfeuchtet sind. Das wird durch eine Typisierung der Standorte von Bülten am Daisetsu San durch Takakura (unveröff.) verdeutlicht (Abb. 4).

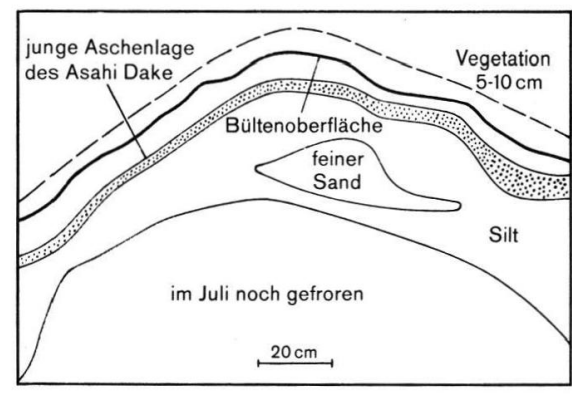

Abb. 3: Querschnitt durch Bülte in Gebiet 2.

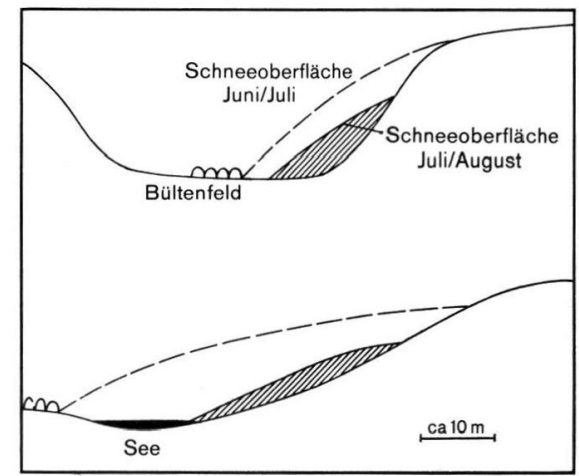

Abb. 4: Typische Standorte von Bültenfeldern nach Y. TAKAKURA (unveröff.) 
Steigt das Gelände an, so werden die Bülten weniger regelmäßig. Bei über $5^{\circ}$ Neigung erheben sich überwachsene Stufen, deren Stirnen mehrere Meter höhenlinienparallel zu verfolgen sind. Bei mehr als $10^{\circ}$ bleiben die TH der Stufen in ähnlicher Weise erhalten, die TF aber sind nur selten noch mit kümmerlichen Pflanzenresten an ihren Rändern ausstaffiert. Der größte Teil ist frei von jedem Bewuchs, und der Schutt wird in der gleichen Weise sortiert, wie es für Gebiet 1 erwähnt wurde. Bei mehr als $20^{\circ}$ bedecken Treppenböden nicht mehr den ganzen Hang; sie sind nur noch als einzelne Kanzeln ausgeprägt (wie auch am Halla San in S-Korea, Ellenberg 1976), dazwischen wachsen Bestände von Pinus pumila. Die Abfolge Bülten - bewachsene Treppenböden - Treppenböden mit vegetationsfreier TF - kanzelartige Treppenböden mit Pinus pumila kann als regelhaft für solche Schneemulden betrachtet werden (Abb. 5). Bei ähnlichen oder gleichen periglazialen Prozessen sind hier zwei Faktoren für die Formausprägung verantwortlich, nämlich die Neigung des Hanges und die Grunddurchfeuchtung.

\section{Gebiet 3: Hokkai Dake}

Vom 2140 m hoch aufragenden Gipfel dachen sich ungleich geformte Hänge ab. Gegen $\mathrm{N}$ und NW sind Steilabstürze vorhanden, die keine Periglazialformen aufweisen. Die anderen Hänge, besonders der in E-Exposition gelegene, sind flach und kaum bewachsen. Pinus pumila fehlt ganz. 90\% des Areals sind hier mit Treppenböden bedeckt. Die TF sind mit scherbigem Schutt gepflastert. In $3-6 \mathrm{~cm}$ Tiefe findet man Grobkomponenten nur noch vereinzelt; sie sind in der relativ jungen Asche des Asahi Dake eingebettet. Die Stirnhöhen der Stufen variieren je nach Hangneigung. Die Stufen zwischen den Flächen mit frostbedingter Sortierung sind dicht bewachsen und zeigen eine mehr oder minder starke Beanspruchung durch Fließbewegungen des Bodens. Zungenartige Girlanden mit Ausbuchtungen bis zu $120 \mathrm{~cm}$ können auftreten. An einigen Stellen hat oberflächlich abfließendes Wasser Breschen durch die Vegetationskanten angelegt und den Schutt der TF durch sie weiter hangabwärts transportiert, so daß auf der ebenen Fläche nur noch randlich größere Steine übrig bleiben, der Rest aber Asche als Oberflächenbedeckung zeigt. Ob solche Stufen völlig aufgefressen werden oder sich regenerieren, ließ sich an dieser Stelle nicht entscheiden. Am SE-Fuß des Hokkai Dake, wo der Hang in eine Verflachung übergeht, findet sich die gleiche Abfolge von Treppenböden über bewachsene Stufen zu Bülten wie sie weiter oben beschrieben wurde. Westlich des Hokkai Dake liegt das Arbeitsgebiet von Омото (1973).

\section{Gebiet 4: Sattel zwischen Hokkai Dake und Hakuun Dake}

Die $1 \mathrm{~km}$ lange und $200-600 \mathrm{~m}$ breite, $2060 \mathrm{~m}$ hohe Sattelfläche weist nur ganz geringe Reliefakzente auf. Sie stellt eine der schönsten Periglazialregionen Japans dar. Pinus pumila ist auf wenige, etwas windgeschützte Mulden beschränkt; der Sattel trägt große Barflächen. Die Schneebedeckung im Winter bleibt infolge heftiger Winde gering oder fehlt ganz (S. Iwata, Mitt.). In dem 2-10 cm hohen Pflanzenteppich sind unregelmäßige Netze von Frostrissen angelegt. Sie spannen drei- bis achteckige Flächen mit Durchmessern bis $\mathrm{zu} 10 \mathrm{~cm}$ auf, die durch schwächer ausgebildete Risse feiner untergliedert werden. Die Flächen zwischen den Rissen können frei von Vegetation sein und Schuttinseln bilden. Die Risse selbst jedoch sind immer Lebensadern und Leitlinien für den Pflanzenwuchs. Sie klaffen an der Oberfläche $0,5-2 \mathrm{~cm}$, in Extremfällen $5 \mathrm{~cm}$ auseinander und verengen sich erst in einer Tiefe von $20 \mathrm{~cm}$. Als Scheideflächen sind sie bis $30 \mathrm{~cm}$ und tiefer verfolgbar. Gegenüber der Umgebung sind die Vegetationsbänder immer leicht abgesenkt, und gröberes Material wird von den Rändern in die Risse hineinverfrachtet. Ob dies durch Frostbewegungen oder Spülprozesse geschieht, spielt keine Rolle. Interessant ist es, daß sich ein einmal gebildeter Frostriß gegenüber den Akkumulationen zu behaupten weiß. Lediglich 
sich stärker entfaltende Pflanzen können die Risse oberflächlich unkenntlich machen. Die Schuttpartien in den Innenflächen setzen sich durch $3-5 \mathrm{~cm}$ hohe Kanten von der Vegetation ab. Diese Ränder zeigen durchweg eine Windbeanspruchung. Das Phänomen des Rasenschälens kann hier genau wie an Treppenböden auftreten.

In 1-2 $\mathrm{m}$ großen, rißumgrenzten Flächen, die nahezu ganz von einem schütteren Pflanzenteppich überkleidet sind, findet man verschiedene Stadien der Aufwölbung. Der Niveauunterschied zwischen der Oberkante der Risse und dem Innenflächen-Mittelpunkt kann 3-20 cm betragen. Dieser Befund, ergänzt durch Beobachtungen an anderen Stellen, läßt eine genetische Erklärung für die Entstehung von Bülten in feinkörnigen und homogenem Substrat zu. Zuerst wird das Rißmuster im Großen angelegt, dann feiner gegliedert. Die an Rissen am besten ausgebildete Pflanzendecke überkleidet dabei die ganze Fläche. Das Tauen des Bodeneises eilt längs der Risse dem übrigen Gebiet voraus; die dadurch entstandene Einmuldung der Stränge verstärkt sich mehr und mehr. Unter den polygonalen Flächen entstehen durch Frostaufwölbungen die Initialformen von Bülten. Freilich müssen nicht alle Bülten durch vorherige Anlage von Rißmustern erklärt werden. Aber hier scheint diese Art der Deutung der Wirklichkeit zu entsprechen.

Bei nicht homogenen und z. T. grobem Substrat und nur lückenhafter Vegetationsbedeckung führt nach KoAzE (1965) der gleiche Vorgang zur Anlage von Steinpolygonen. Beim Tauen verlagern sich besonders die größeren Steine von der Aufwölbung zu den Rissen hin. Diese werden aber nur saisonal gefüllt und klaffen in jedem Jahr weiter auf. Bei jedem Auffriervorgang wird der nach und nach feinkörniger gewordene und somit feuchtere Innenteil stärker betroffen. Bei jedem Tauen kann sich eine weitere Scheidung der verschiedenen Komponenten ergeben.

Im Gebiet 4 können Formen der verschiedensten Entwicklungsstdien dieser Steinpolygone gefunden werden. Einige mit Durchmessern zwischen 50 und $180 \mathrm{~cm}$ sind dabei vollkommen entwickelt; d. h. weitere Frost-Tau-Zyklen können die Trennung der Komponenten nicht mehr verstärken (Taf. I, Fig. 2). Die Innenflächen der Polygone wirken feucht; die verwitterte Asche wird nur dünn von Bruchstücken bedeckt, die nicht größer als $1-2 \mathrm{~cm}$ sind. Der bis $30 \mathrm{~cm}$ breite Rahmen enthält alle größeren Fraktionen zwischen 1 und $15 \mathrm{~cm}$. Die Tiefe der Sortierung im Rahmen überschreitet $20 \mathrm{~cm}$ nicht. Bei kaum merkbarer Neigung verzerren sich die Polygone in Fallinie. Schon bei $5^{\circ}$ sind Steinstrei-

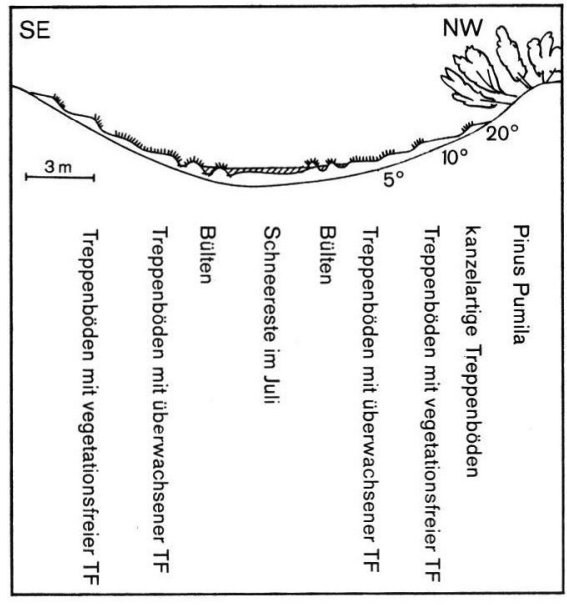

Abb. 5: Schematischer Querschnitt durch eine Schneemulde.

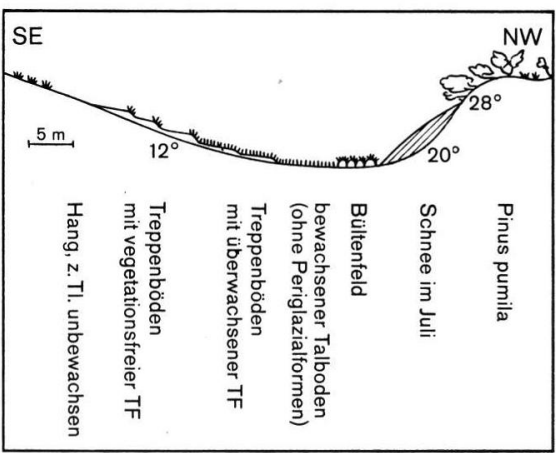

Abb. 6: Schematischer Querschnitt durch ein asymmetrisches Tal. 
fen deutlich ausgebildet. Sie liegen nicht streng parallel, sondern laufen auseinander und vereinigen sich, indem sie kleine Vegetationsinseln umfließen.

Vom Sattel gehen einige Talmulden aus. Ihre Anfänge sind durch eine deutliche Ausprägung primärer Hangasymmetrie gekennzeichnet (Taf. I, Fig. 3). Als Beispiel dafür mag eine gegen SW abfallende Talmulde gelten (Abb.6). Ihr rechter, nach SE ausgerichteter Hang ist zwischen 20 und $30^{\circ}$ steil; angewehter Schnee bleibt dort bis in den Juli hinein liegen. Am Hangfuß sind Bülten ausgebildet. Die gegenüberliegende, meist auch im Winter apere Seite wird nicht steiler als $15^{\circ}$. Auf ihr erscheinen Treppenböden, die im flacheren Bereich ganz, im steileren nur am TH bewachsen sind. Der Boden der Talmulde ist bewachsen und frei von Periglazialformen.

Am S-Ende des Sattels, am $20-30^{\circ}$ steilen Abhang des Hakuun Dake, sind Halden mit Blöcken bis zu $2 \mathrm{~m}$ Durchmesser ausgebildet. Einige formen Blockströme mit mächtigen Zungen. Rezente Bewegungen sind festzustellen. An zwei Zungen wurden Stauchwülste bis zu $80 \mathrm{~cm}$ aufgeschoben (Taf. I, Fig. 4). Im Innern der Blockströme müssen Eisfüllungen vorhanden sein, denn an vielen Stellen drang Wasser hervor, obwohl der Schnee bereits geschmolzen war.

\section{Gebiet $5: \mathrm{Haku}$ n Dake und Umgebung}

Der Hakuun Dake $(2230 \mathrm{~m})$ ist die höchste Erhebung eines Kraters, der einen flachen, mit feinem Material angereicherten Kratergrund in $2170 \mathrm{~m}$ Höhe umschließt. Frostwechselbedingte Sortierungen konnten dort nur an den Rändern entstehen, wo gröberes $\mathrm{Ma}$ terial eingespült wurde. Einige schlecht entwickelte Steinpolygone sind auf vegetationsfreien Flächen zu finden. Der Großteil des Grundes ist ein Bültenfeld von ca. $250 \mathrm{~m}$ Durchmesser. Die bis $60 \mathrm{~cm}$ hohen Aufwölbungen sind fast alle bewachsen (Taf. II, Fig. 1). Stellenweise ist in den Rinnen zwischen den Bülten ein polygonales Rißnetz zu erkennen, was T. KoAze (Mitt.) veranlaßte, die Entstehung dieser Bülten als aufgepreßte Mittelteile der Innenflächen in einem Frostriß-Muster zu erklären.

An der E-Abdachung des Kraters liegen einige sanft geböschte vegetationsarme Hangpartien und Verflachungen. Hier sind Steinpolygone mit vollständiger Sortierung ausgebildet; ihre Durchmesser betragen bis zu $150 \mathrm{~cm}$. Grobmaterial über $5 \mathrm{~cm}$ Durchmesser ist auf den Innenflächen nicht zu finden. In den Rahmen wurde Grobmaterial bis in $12 \mathrm{~cm}$ Tiefe angereichert. An den Hängen gehen die Polygone in weitständige Steinstreifen über. Bei diesen Formen ist das Grobmaterial oberflächlich mit Flechten überzogen; eine allwinterliche Bewegung dieser Komponenten ist deshalb als unwahrscheinlich anzusehen.

Interessant ist eine $2160 \mathrm{~m}$ hohe Kuppe im NE des Hakuun Dake. Allseitig dacht sie sich flach ab; die Hänge sind durch 3-10 m tiefe Talmulden gegliedert. Diese waren z. T. noch schneegefüllt und gaben an ihren Rändern Bültenfelder frei, die bei stärkerer Neigung in Treppenböden übergehen. Girlanden besetzen die Hangfläche überall, oft mit schütter bewachsenen Stufenstirnen, oft als gänzlich vegetationsfreie Schutt-Treppen (Taf. II, Fig. 2). In horizontaler Lage haben sich bis $50 \mathrm{~cm}$ große Feinmaterial-Inseln gebildet, die an vergrößerte Erdknospen erinnern (z. B. Furrer \& Freund 1973). Im September 1971 brachte M. Fukuda (Mitt.) einige Handbohrungen an und stieß auf Permafrost. Der frost-table lag durchschnittlich in $160 \mathrm{~cm}$ Tiefe. Auf die Periglazialformen hat der dauernd gefrorene Untergrund keinen Einfluß, da das frei werdende Wasser seitlich abfließen kann. Ein Beweis für das Perennieren von Bodeneis wurde in Japan davor nur am Hauptkrater des Fuji San erbracht (Higuchi \& FujII 1971). 
Gebiet 6: Hakuun Dake bis Chûbetsu Dake

Zwischen Hakuun Dake und Tomuraushi Yama sind Periglazialformen seltener. Zwar herrschen flache Hangneigungen weiterhin vor, doch gewinnt die Vegetation mehr an Bedeutung. Sasa, allein oder im Verband mit Pinus pumila, nimmt große Bereiche ein. Die Latschen zeigen häufig eine vom sommerlichen Wind aus SW deformierte Wuchsform. Schneedruckspuren können nur lokal festgestellt werden. Stellenweise finden sich Muster, die an Treppenböden erinnern. Es sind $30-70 \mathrm{~cm}$ breite Schuttstreifen, die durch $20-40$ $\mathrm{cm}$ breite Vegetationsbänder getrennt sind. Doch fehlen Höhenunterschiede zwischen nackten und bewachsenen Flächen. Die Musterung verläuft von SW nach NE, entsprechend der vorherrschenden Sommer-Windrichtung. Rasenschälen, bedingt durch Wind unter Mithilfe von Kammeis beansprucht die Vegetationsränder. Unter den nackten und bewachsenen Flächen weisen die Komponentengrößen des Materials keine signifikanten Unterschiede auf. Dünne Feinmaterial-Anreicherungen in den Vegetationsstreifen sind wohl eher auf Pflanzenwuchs als auf eine frostbedingte Sortierung zurückzuführen. Abstufungen zwischen diesen Vegetationsstreifen und den Treppenböden sind zahlreich ausgebildet.

Treppenböden großen Ausmaßes, d. h. mit bis zu $150 \mathrm{~cm}$ breiten Flächen, die am Hangfuß in bewachsene Stufen und Bülten übergehen, konnte ich lediglich am W-Hang über einem kleinen Bergsee feststellen. Nördlich von ihm liegen am Fuß einer $60 \mathrm{~m}$ hohen Wand in E-Exposition bei $1750 \mathrm{~m}$ Blöcke, die von Schneeflecken umgeben sind und sich etwas von der Schuttschleppe des Hangfußes absetzen. Die Blöcke haben etwa $30 \mathrm{~cm}$ bis $2 \mathrm{~m}$ Durchmesser und sind so angeordnet, daß 3-6 m messende Innenflächen blockarm bleiben. Von T. Koaze (Mitt.) wird dieser Befund als fossiles Steinnetzwerk gedeutet. TAKaKura (1972) beschreibt die Formen und vergleicht sie mit kleinen rezenten Steinpolygonen am Ontake und Norikura Dake. Meines Erachtens ist es spekulativ, in diesen undeutlich sortierten Blockringen Reste eines fossilen Periglazial-Formenschatzes zu sehen. Ich halte sie eher für zufällige Bildungen.

Beim Chûbetsu Dake $(1963 \mathrm{~m})$ sind in E-Exposition an Hängen bis zu $20^{\circ}$ Neigung große Treppenböden ausgebildet. Auf der Kuppe initiieren kleine Rißmuster mit einer Netzweite von $10-20 \mathrm{~cm}$ Sortierungserscheinungen. Die Risse sind mit Bruchstücken bis 15 mm Größe gefüllt; auf den Innenflächen liegen kleinere Komponenten und herrscht verwitterte Asche vor.

Gebiet 7 : Bergsee $2 \mathrm{~km}$ südöst 1 ich des Kaun Dake

Am flachen E-Ufer des Bergsees, wo der Grund erst im Spätsommer trocken fällt, sind Steinnetze mit Maschenweiten zwischen 1 und $2 \mathrm{~m}$ ausgebildet. Ende Juli lagen sie noch $40 \mathrm{~cm}$ unter Wasser.

Tafe I

Fig. 1: Gebiet 2, $1780 \mathrm{~m}$, Schneemulde mit Bültenfeld, an flacheren Partien des südl. exponierten Hanges stellenweise Treppenböden, an steileren dichter Bewuchs mit Pinus pumila. Aufnahme Ende Juli 1973. - Fig. 2: Gebiet 4, $2060 \mathrm{~m}$, gut sortierte Steinpolygone mit feuchter Innenfläche. Fig. 3: Gebiet 4, Standort bei $2060 \mathrm{~m}$, Blick gegen SW. Ausbildung primärer Hangasymmetrie in Talmulde. - Fig. 4: Gebiet 4, 2080 m, Stauchwulst unter Blockhalte. 

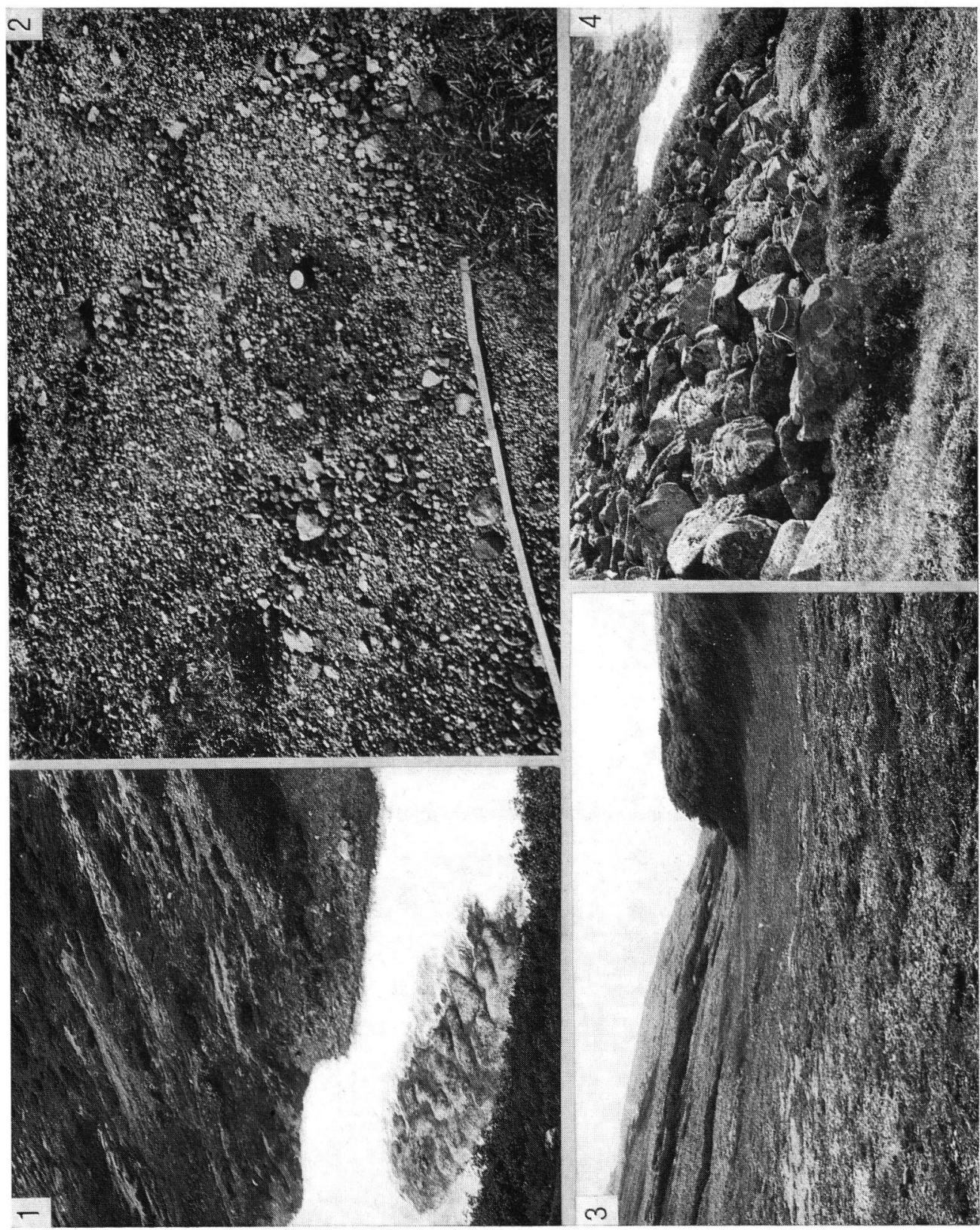
Gebiet 8: Tomuraushi Yama

Der bis $2141 \mathrm{~m}$ aufragende Gipfelstock ist mit Trümmern überkleidet; Periglazialformen sind nicht ausgebildet.

Auf feuchten Verflachungen im N des Gipfels finden sich Bültenfelder. Hier sind auch die angeblich schönsten Periglazialformen Japans zu finden: $1-2 \mathrm{~m}$ große Steinpolygonnetze mit vollkommener Sortierung. Sie bleiben ähnlich wie in Gebiet 7 bis in den August hinein wasserbedeckt (SAко et al., zitiert nach Омото 1973).

Südlich des Tomuraushi Yamas sind an windgefegten Gratlagen Treppenböden zu finden. Unterhalb $1620 \mathrm{~m}$ wachsen schneegedrückte Betula-Bestände, und die Vegetation der alpinen Stufe klingt aus. In den geschützten Tallagen werden nahezu alle Hänge von Wald und Gebüsch eingenommen.

\section{Zur Regelhaftigkeit des Auftretens und der Vergesellschaftung von Periglazialformen}

Es wurden hier ausschließlich Periglazialformen beschrieben, die über der Waldgrenze liegen. Dort finden sich die meisten, aber nicht alle (Ellenberg 1974). Von 91 Arealen mit Periglazialfunden in Zentral-Hokkaido liegen nach Angaben von KoAze (1958, 1965)

$\begin{array}{lr}\text { über } 2150 \mathrm{~m} & 9 \% \\ \text { zwischen } 2050 \text { und } 2150 \mathrm{~m} & 18 \% \\ \text { zwischen } 1950 \text { und } 2050 \mathrm{~m} & 27 \% \\ \text { zwischen } 1850 \text { und } 1950 \mathrm{~m} & 31 \% \\ \text { unter } 1850 \mathrm{~m} & 15 \%\end{array}$

Das häufige Auftreten der Formen zwischen 1850 und $2050 \mathrm{~m}(58 \%)$ bedeutet kein klimatisch zu erklärendes Optimum. In dieser Höhe sind lediglich große Gebiete mit flachen Neigungen und schütterer Pflanzendecke vorhanden. Die Areale darüber sind genauso gut geeignet; nur sind sie flächenmäßig weniger stark vertreten und häufig steiler geböscht. Die Areale darunter sind im Gebiet des Daisetsu San meist steil und mit dichter Vegetation überkleidet. Kälteperioden mit genügend häufigen Frostwechseln kommen aber auch dort vor. Wo die Vegetation der Solifluktion kein zu großes Hindernis entgegensetzt, sind Periglazialformen auch weit unterhalb der klimatischen Waldgrenze ausgebildet, und zwar nicht nur Bülten, die im Tiefland von Konzen in NE-Hokkaido rezente Aktivität anzeigen (S. Iwata, Mitt.), sondern auch Terrassenböden, Steinstreifen und -polygone.

Schlüsse über die untere Begrenzung der Periglazialstufe werden mit den skizzierten Beobachtungen im Gebiet des Daisetsu San nicht angestrebt. Hingegen lassen sich Aussagen über die Regelhaftigkeit des Auftretens von Periglazialformen und ihrer Vergesellschaftung machen.

Tafel II

Fig. 1: Gebiet 5, Bültenfeld, großenteils bewachsen. Rasenkliffs im Mittelgrund durch sommerlichen Windeinfluß aus S und SW bedingt. - Fig. 2: Gebiet 5, Standort $2150 \mathrm{~m}$, östl. Hakuun Dake. Girlandenförmig ausgebildete Treppenböden, hier über Permafrost. 


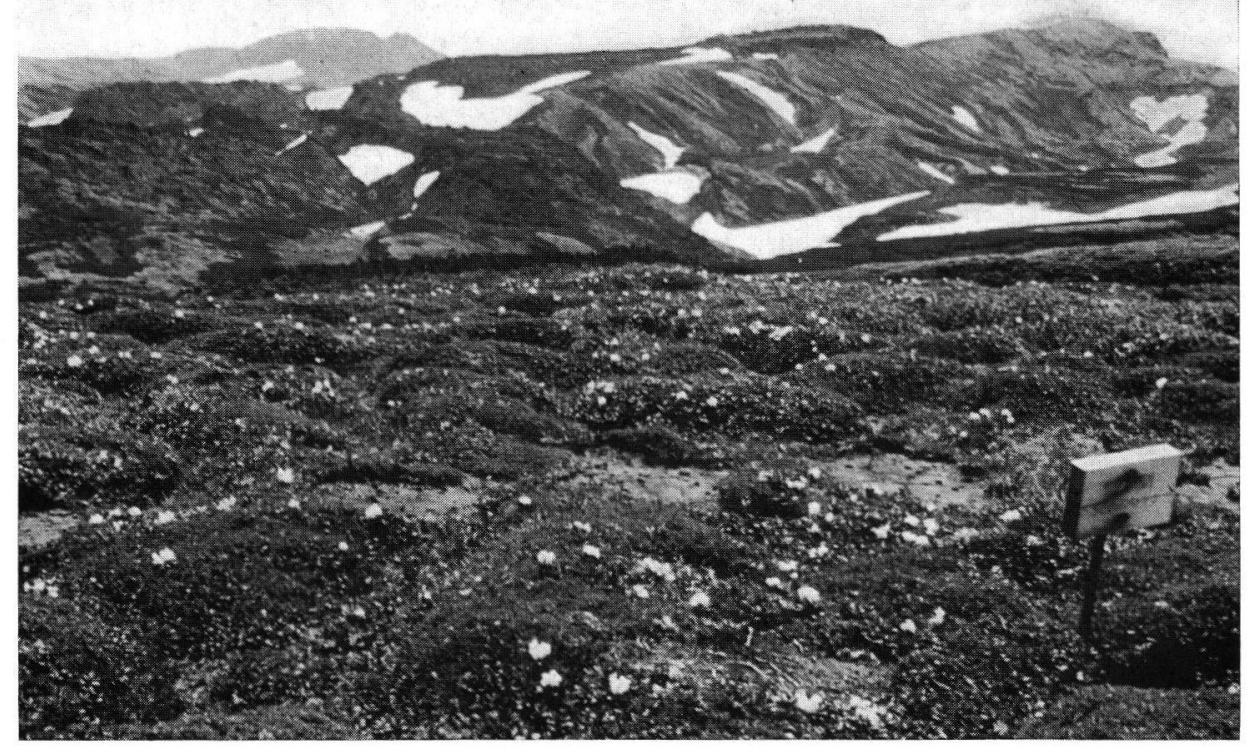

Fig. 1

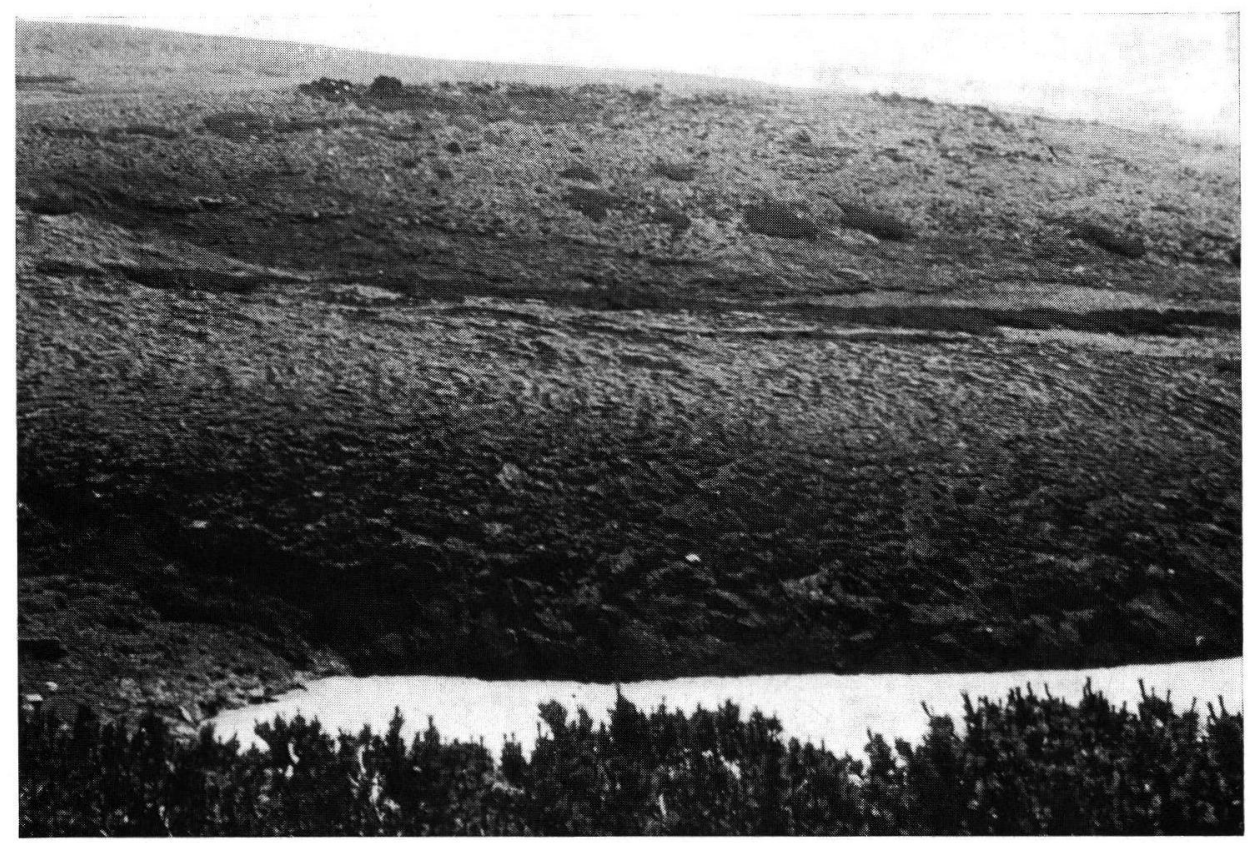

Fig. 2 
Die meisten Fundareale liegen im Bereich breiter, sanft abfallender Grate. Der Vertikalabstand zu den Graten beträgt bei

$82 \%$ der Fundareale weniger als $25 \mathrm{~m}$

$4 \%$ der Fundareale zwischen 25 und $60 \mathrm{~m}$

$5 \%$ der Fundareale zwischen 60 und $100 \mathrm{~m}$

$8 \%$ der Fundareale mehr als $100 \mathrm{~m}$.

Der Klassifikation von WASHBURN (1956) folgend, entfallen von den Funden auf nonsorted steps $32 \%$

sorted stripes

$23 \%$ (die Hälfte davon mit weitem Abstand)

sorted polygons

$20 \%$ (davon zwei Drittel Großformen)

nonsorted polygons

sorted steps

$12 \%$ (alles Großformen)

andere

$7 \%$

$6 \%$ (nons. stripes, sorted und nons. circles)

Keines der Fundareale ist im Sommer ein trockener Standort. Nach dem Grad der Bodenfeuchte wurden die Areale wie folgt gruppiert:

$\begin{array}{lr}\text { dry } & 0 \% \\ \text { moderate wet } & 63 \% \\ \text { wet } & 23 \% \\ \text { water stagnated } & 14 \%\end{array}$

Die Periglazialformen sind mithin zu $53 \%$ an Standorte gebunden, die weniger als $6^{\circ}$ geböscht sind. Nur $13 \%$ sind an Hängen mit mehr als $14^{\circ}$ Neigung ausgebildet. Im Gegensatz zu den Japanischen Alpen, wo vor allem die W-Seite Periglazialformen aufweist und die E-Seite fast frei davon ist, spielt die Exposition beim Daisetsu San eine sehr untergeordnete Rolle, wie aus Abb. 7 ersichtlich ist. Nach KOAZE (1965) waren 80\% seiner Funde windexponiert. Daneben lagen $15 \%$ in Schneemulden oder Kraterlöchern und nur $5 \%$ an anderen Stellen. Seine Untersuchungen und meine ergänzenden Beobachtungen erlauben es, für das Gebiet des Daisetsu San ein Schema aufzustellen, das die Ausbildung von Periglazialformen über der Waldgrenze in Abhängigkeit von Relief, Windeinfluß, Schneeverhältnissen und Pflanzendecke verdeutlicht (Tab. 1).

\section{Zusammenfassung}

$\mathrm{Da}$ in Japan nur kleine Areale über die Waldgrenze aufragen und diese arm sind an Ebenheiten und flachen Hängen, gibt es nur sehr eng umgrenzte Bereiche mit aktiver periglazialer Formung. Die Ungunst für die Ausprägung solcher Formen wird durch das Vorherrschen vulkanischen Lockermaterials und gebietsweise durch lang dauernde und hohe Schneebedeckung verstärkt.

Die Vulkangruppe des Daisetsu San stellt demgegenüber eine Ausnahme dar. In diesem, in Zentral-Hokkaido gelegenen Berggebiet ist ein vielfältiger periglazialer Formenschatz auf großen Flächen zu studieren. Treppenböden verschiedener Art, Bültenfelder, Steinpolygone und -streifen meist großen Ausmaßes herrschen vor. Diese Formen sind nicht an Permafrost gebunden, wohl aber an geeignetes Bodenmaterial (andesitischer Schutt in verwitterter Asche), flache Hangneigung, Vegetationsarmut und - außer bei Bülten - geringe Schneebedeckung.

Zusammen mit den Beobachtungen japanischer Autoren läßt sich ein Schema aufstellen, das die Regelhaftigkeit des Auftretens von Periglazialformen und ihrer Vergesellschaftung zeigt (Tab. 1). 


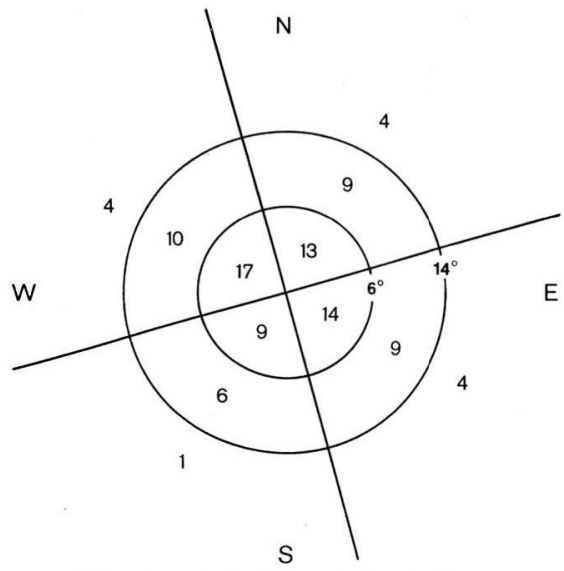

$53 \%$ der Funde an Hängen flacher als $6^{\circ}$

$34 \%$ der Funde an Hängen mit 6-14 Neigung

$13^{\circ} \%$ der Funde an Hängen steiler als $14^{\circ}$

Abb. 7: Gliederung der Funde nach Exposition und Hangneigung.

Tab. 1: Die Regelhaftigkeit des Auftretens von Periglazialformen und ihrer Vergesellschaftung in Gebiet des Daisetsu San

\begin{tabular}{l|l|l}
\hline Relief & $\begin{array}{l}\text { Windverhältnisse, Schneebedeckung, } \\
\text { Vegetation }\end{array}$ & Ausbildung von Periglazialformen \\
\hline $\begin{array}{lll}\text { Kuppen wie } \\
\text { Gebiet 1 }\end{array}$ & $\begin{array}{c}\text { außer im winterlichen Lee } \\
\text { mit Vegetation bedeckt. Im allg. } \\
\text { durch Wind beeinflußt und keine große Ak- } \\
\text { kumulation von Schnee. Pinus pumila } \\
\text { zurücktretend }\end{array}$ & $\begin{array}{c}\text { Sortierungserscheinungen auf ebenen und } \\
\text { leicht geböschten Partien häufig } \\
\text { flähenhaftes Auftreten von Treppenböden im } \\
\text { Kuppengebiet und an allen leicht gebösch- } \\
\text { ten Hängen. Auf Verflachungen Ubergang } \\
\text { zu Bülten }\end{array}$ \\
\hline
\end{tabular}

\begin{tabular}{|c|c|c|}
\hline \multirow{2}{*}{$\begin{array}{l}\text { Breite Grate } \\
\text { und } \\
\text { flache Hänge } \\
\text { wie Gebiet } 3\end{array}$} & $\begin{array}{l}\text { bei (sommerlichem) Windeinfluß fast kein } \\
\text { Aufkommen von deckender Vegetation. } \\
\text { Schneedecke meist nur dünn }\end{array}$ & $\begin{array}{l}\text { flächenhaftes Auftreten von Treppenböden } \\
\text { und Steinstreifen }\end{array}$ \\
\hline & $\begin{array}{l}\text { bei fehlendem Windeinfluß oft deckend mit } \\
\text { Pinus pumila bestanden }\end{array}$ & kaum Periglazialformen \\
\hline \multirow[t]{2}{*}{$\begin{array}{l}\text { Sattelflächen } \\
\text { wie Gebiet } 4\end{array}$} & $\begin{array}{l}\text { größtenteils stark windexponiert, deshalb } \\
\text { vegetationsarm und im Winter kaum } \\
\text { schneebedeckt }\end{array}$ & $\begin{array}{l}\text { Frostrisse, Steinpolygone, Vegetationsbän- } \\
\text { der, stellenweise Bültenfelder }\end{array}$ \\
\hline & $\begin{array}{l}\text { in windgeschützten Lagen stellenweise mit } \\
\text { Pinus pumila bestanden }\end{array}$ & keine Periglazialformen außer Frostrissen \\
\hline $\begin{array}{l}\text { Schneemulden } \\
\text { wie Gebiet } 2\end{array}$ & $\begin{array}{l}\text { reduzierte Vegetationsbedeckung wegen } \\
\text { starker Schneeakkumulation }\end{array}$ & $\begin{array}{l}\text { Bültenfelder im engeren Bereich der Schnee- } \\
\text { mulden, Terrassetten an den flachen Hän- } \\
\text { gen }\end{array}$ \\
\hline $\begin{array}{l}\text { Kraterlöcher } \\
\text { wie im Gebiet } 5\end{array}$ & $\begin{array}{l}\text { meist reduzierte Vegetation wegen langer } \\
\text { Dauer der Schneebedeckung }\end{array}$ & $\begin{array}{l}\text { Bültenfelder im Kratergrund, Steinstreifen } \\
\text { und Treppenböden an den Ubergängen zur } \\
\text { Kraterwand }\end{array}$ \\
\hline $\begin{array}{l}\text { Asymmetrische } \\
\text { Talmulden } \\
\text { wie im Gebiet } 4\end{array}$ & $\begin{array}{l}\text { im winterlichen Lee lange Schnee, ebenso } \\
\text { im Talgrund. Am Luvhang keine Schnee- } \\
\text { akkumulation. Vegetation reduziert, Pinus } \\
\text { pumila nur stellenweise am Steilhang }\end{array}$ & $\begin{array}{l}\text { Bülten am Hangfuß, sofern er lange schnee- } \\
\text { bedeckt bleibt. Keine Periglazialformen im } \\
\text { Talgrund. Treppenböden am wenig bewach- } \\
\text { senen flachen Hang }\end{array}$ \\
\hline Steilabstürze & $\begin{array}{l}\text { extrem vegetationsarm und dauernd schnee- } \\
\text { frei }\end{array}$ & $\begin{array}{l}\text { intensive Frostsprengung und Bildung von } \\
\text { Blockhalden am Fuß der Abstürze }\end{array}$ \\
\hline $\begin{array}{l}\text { Felsburgen } \\
\text { wie im Gebiet } 8\end{array}$ & $\begin{array}{l}\text { vegetationsarm, an winterlich windexponier- } \\
\text { ten Seiten dauernd schneearm }\end{array}$ & $\begin{array}{l}\text { keine Periglazialformen, weil zu steil und } \\
\text { Material zu grobblockig, Produktion von } \\
\text { Frostschutt, evtl. Bildung von Blockströ- } \\
\text { men. }\end{array}$ \\
\hline
\end{tabular}




\section{Schriftenverzeichnis}

Ellenberg, L. (1974): The periglacial stage in Europe (especially the Alps and Japan - a comparison. - Geogr. Rep. of Tokyo Metropolitan University, 9: 53-65; Tokyo.

- (1976): Rezente Periglazialerscheinungen auf Cheju-Do, Südkorea. — Geogr. Helv., 2: 69-74; Bern.

Furrer, G. \& Freund, R. (1973): Beobachtungen zum subnivalen Formenschatz am Kilimandjaro. - Z. Geomorph. N. F., Suppl. Bd. 16: 180-203; Berlin.

Harris, C. (1974): Autumn, winter and spring soil temperatures in Okstindan, Norway. - J. of Glaciology, 13/69: 521-533; Cambridge.

Higuchi, K. \& FujII, Y. (1971): Permafrost at the summit of Mount Fuji, Japan. - Nature, 230/ 5295: 521 only; London.

Koaze, T. (1958): Verbreitung rezenter und fossiler Periglazialerscheinungen in Japan (übersetzter Titel, japanisch, Manuskript). - Meiji Univ.; Tokyo.

- (1965): The patterned grounds on the Daisetsu Volcanic Group, Central Hokkaido. - The Geogr. Rev. of Japan, 38/3: 179-199; Tokyo.

Numata, M. (1972): Ecological interpretations of vegetational zonation of high mountains, particulary in Japan and Taiwan. - Erdwiss. Forsch., 4: 288-299; Wiesbaden.

Омото, K. (1973): Recent polygons in Daisetsu-zan National Park, Hokkaido. - The Sci. Rep. of Tohoku Univ., 7 th Ser., 23/1: 29-38.

Schwind, M. (1967): Das Japanische Inselreich, Bd. 1, Die Naturlandschaft. - Berlin.

TAKakura, Y. (unveröff.): Bülten im Gebiet des Daisetsu San (übersetzter Titel, japanisch, Manuskript); Obihiro, Japan.

- (1972): Debris Islands of Daisetsu San. - Chikyû Kogatsu (Earth Sci.), 26/3: 134-135 (japanisch); Tokyo.

WASHBURN, A. L. (1956): Classification of patterned grounds and review of suggested origins. Bull. Geol. Soc. America, 67/7: 823-865; Baltimore.

Weltforstatlas - Bundesforschungsanstalt für Forst- und Holzwirtschaft, Reinbek b. Hamburg [Hrsg.]; Berlin 1973.

Manuskript eingeg. 22. 10. 1976. 